\title{
Morphologic changes in boar sperm nuclei with reduced disulfide bonds in electrostimulated porcine oocytes
}

\author{
Michiko Nakai ${ }^{1,2}$, Naomi Kashiwazaki², Akiko Takizawa², Naoki Maedomari ${ }^{1,2}$, \\ Manabu Ozawa ${ }^{1}$, Junko Noguchi ${ }^{1}$, Hiroyuki Kaneko ${ }^{1}$, Masao Shino ${ }^{2}$ and Kazuhiro Kikuchi ${ }^{1}$ \\ ${ }^{1}$ Genetic Diversity Department, National Institute of Agrobiological Sciences, Kannondai 2-1-2, Tsukuba, Ibaraki \\ 305-8602, Japan and ${ }^{2}$ Laboratory of Animal Reproduction, School of Veterinary Medicine, Azabu University, \\ Sagamihara, Kanagawa 229-8501, Japan \\ Correspondence should be addressed to K Kikuchi; Email: kiku@nias.affrc.go.jp
}

\begin{abstract}
In pigs, failure of sperm nuclear decondensation has been reported after injection into oocytes. We examined the effects of pretreating sperm heads with Triton X-100 (TX-100) and dithiothreitol (DTT) and of electrical stimulation of oocytes after sperm head injection on time-dependent morphologic changes in sperm nuclei and in vitro development to the blastocyst stage. In experiment 1, spermatozoa were pretreated with $1 \%$ TX-100 and $5 \mathrm{mM}$ DTT (T + D) or not treated, and then injected into in vitro matured oocytes. Electrical stimulation $(1.5 \mathrm{kV} / \mathrm{cm}, 20 \mu \mathrm{sC}$ pulse) was applied to the oocytes $1 \mathrm{~h}$ after injection (stimulated group) or was not applied (unstimulated group). Some of the oocytes in each group were evaluated at hourly intervals until $10 \mathrm{~h}$ after injection for morphologic changes in the sperm nuclei. Unstimulated oocytes injected with untreated spermatozoa showed a delayed peak in the rate of nuclear decondensation (39.4-44.1\%, 3-6 $\mathrm{h}$ after injection) compared with oocytes injected with T + D-treated spermatozoa $(57.0 \%$ and $52.6 \%, 1$ and $2 \mathrm{~h}$, respectively). The rate of male pronucleus formation peaked $6 \mathrm{~h}$ after stimulation (by 40-60\%) after injected oocytes had been stimulated with an electrical pulse, irrespective of whether or not the spermatozoa had been pretreated. In unstimulated oocytes, the rate of male pronucleus formation did not increase and stayed at the basal level (less than $20 \%$ ) throughout the culture period, regardless of the sperm treatment. Thus, $T+D$ treatment of spermatozoa did not affect completion of fertilization. In experiment 2 , we evaluated the effects of electrical stimulation and sperm treatment with $T+D$ on the rate of blastocyst formation and the mean number of cells per blastocyst. Oocytes stimulated after injection with either T + D-treated or untreated spermatozoa showed significantly higher percentages of blastocyst formation $(24.8 \%$ and $27.1 \%$ respectively) than did unstimulated oocytes $(1.1 \%$ and $4.1 \%$ for $T+D$-treated and untreated respectively; $P<0.01$ by Duncan's multiple-range test). The rate of blastocyst formation did not differ between the $T+D$-treated and untreated groups. The mean number of cells per blastocyst did not differ among any of the groups (14.0-29.4 cells). These results suggest that pretreatment of sperm with TX-100 and DTT shifted the timing of sperm nuclear decondensation forward. However, pronucleus formation and development to the blastocyst stage in vitro were not improved by sperm treatment. Thus, electrical stimulation of injected oocytes enhances in vitro development to the blastocyst stage in pigs.

Reproduction (2006) 131 603-611
\end{abstract}

\section{Introduction}

Intracytoplasmic injection of a nonmotile sperm into the ooplasm for assisted fertilization is a highly specialized procedure for producing new livestock, and is also expected to be useful for sperm-mediated gene transfer (Perry et al. 1999). Although successful production of viable piglets by intracytoplasmic sperm injection (ICSI), using both in vivo (Kolbe \& Holtz 2000, Martin 2000) and in vitro (Nakai et al. 2003) matured oocytes, has been reported, these methods have low successful fertilization rates (Nakai et al. 2003). In many injected pig oocytes, the female pronucleus forms, but most of the sperm heads do not decondense (Kren et al. 2003). The injected sperm nuclei have to be remodeled into the typical somatic state, and participate in subsequent chromosomal activities such as nuclear decondensation and formation of male pronuclei. Remodeling of sperm nuclei requires the reduction of disulfide bonds $(\mathrm{S}-\mathrm{S})$ regulated by ooplasmic glutathione (Perreault et al. 1988, Zirkin et al. 1989, Yoshida et al. 1993, Katagiri \& Ohsumi 1994) and replacement of protamines by histones (Yanagimachi 1994). These events are considered essential for the completion 
of fertilization (Shimada et al. 2000). If it were possible to regulate the $S-S$ status of sperm nuclei before injection, the relationship between $S-S$ reduction and sperm decondensation or pronucleus formation would be clearer, and the efficacy of porcine ICSI might be improved. Dithiothreitol (DTT) is a common chemical agent used to reduce sperm nuclear $\mathrm{S}-\mathrm{S}$, resulting in artificial decondensation in vitro in mammalian spermatozoa (Seligman et al. 1994, Motoishi et al. 1996, Rho et al. 1998, Galli et al. 2003), including those of pigs (Shimada et al. 2000). It has also been reported that in hamsters there is a significant relationship between $\mathrm{S}-\mathrm{S}$ reduction by DTT and pronucleus formation (Perreault et al. 1987). We have already developed an ICSI technique that achieves developmental competence of embryos, resulting in the production of viable piglets after embryo transfer (Nakai et al. 2003). Use of a combination of DTT treatment and the ICSI technique would enable us to clarify the influence of the process of $\mathrm{S}-\mathrm{S}$ reduction on decondensation and pronucleus formation and, furthermore, its influence on development to the blastocyst stage after injection.

On the other hand, oocyte activation is also an event fundamental to embryonic development in ICSI. In mice (Kimura \& Yanagimachi 1995, Kuretake et al. 1996), hamsters (Hoshi et al. 1992), man (Tesarik \& Sousa 1995) and rabbits (Keefer 1989), ICSI alone is sufficient to activate oocytes for embryonic development. However, in pigs, artificial oocyte activation is considered essential for successful ICSI; the additional electrical stimulation after ICSI results in better embryonic development than after injection alone (Lee et al. 2003). It has also been reported that oocyte activation by electrical stimulation enhances normal fertilization after injection with round spermatids (Kim et al. 1999) and the development of oocytes to the blastocyst stage after ICSI with frozen-thawed sperm (Nagashima et al. 2003). However, the precise effect of oocyte stimulation on embryonic development, in relation to sperm decondensation, has not yet been clarified.

We examined the effects of pretreating sperm with Triton X-100 (TX-100) and DTT, and also of electrical stimulation of oocytes, on time-dependent morphologic changes in the sperm nucleus and on in vitro development to the blastocyst stage.

\section{Materials and Methods}

\section{Oocyte collection and in vitro maturation}

Protocols for the use of animals were approved by the Animal Care Committee of the National Institute of Agrobiological Sciences and Azabu University, Japan. Ovaries were obtained from prepubertal cross-bred gilts (Landrace, Large White, and Duroc breeds) at a local slaughterhouse and transported to the laboratory at $35^{\circ} \mathrm{C}$. Cumulusoocyte complexes (COCs) were collected from follicles 2-6 mm in diameter in TCM 199 (with Hanks' salts; Sigma) supplemented with $10 \%(\mathrm{v} / \mathrm{v})$ fetal bovine serum
(Gibco; Invitrogen Corp., Carlsbad, CA, USA), $20 \mathrm{mM}$ Hepes (Dojindo Laboratories, Kumamoto, Japan), $100 \mathrm{IU} / \mathrm{ml}$ penicillin G potassium (Sigma), and $0.1 \mathrm{mg} / \mathrm{ml}$ streptomycin sulfate (Sigma). Maturation culture was performed as reported previously (Kikuchi et al. 2002, Nakai et al. 2003). In brief, about 40 COCs were cultured in four-well dishes (Nunclon Multidishes; Nalge Nunc International, Rochester, NY, USA) for $20-22 \mathrm{~h}$ in $500 \mu \mathrm{l}$ maturation medium, which was a modified North Carolina State University (NCSU)-37 solution (Petters \& Wells 1993) containing $10 \%(\mathrm{v} / \mathrm{v})$ porcine follicular fluid, $0.6 \mathrm{mM}$ cysteine, $50 \mu \mathrm{M} \beta$-mercaptoethanol, $1 \mathrm{mM}$ dibutyl cAMP (dbcAMP; Sigma), $10 \mathrm{IU} / \mathrm{ml}$ equine chorionic gonadotropin (PMS 1000 Tani NZ; Nihon Zenyaku Kogyo, Koriyama, Japan), and $10 \mathrm{lU} / \mathrm{ml}$ human chorionic gonadotropin (Puberogen $1500 \mathrm{U}$; Sankyo, Tokyo, Japan). This was followed by culture for a further $24 \mathrm{~h}$ in maturation medium without dbcAMP and hormones. Maturation culture was carried out at $39^{\circ} \mathrm{C}$ in an atmosphere of $\mathrm{CO}_{2}, \mathrm{O}_{2}$ and $\mathrm{N}_{2}$ adjusted to $5 \%, 5 \%$ and $90 \%$ respectively. After maturation culture, cumulus cells were removed from the oocytes by treatment with $150 \mathrm{IU} / \mathrm{ml}$ hyaluronidase (Sigma) and gentle pipetting. Denuded oocytes with the first polar body were harvested under a stereomicroscope and served as in vitro matured oocytes.

\section{Sperm preparation and pretreatment}

Spermatozoa for injection were prepared as reported previously (Nakai et al. 2003). Epididymal spermatozoa were collected from a Landrace boar and frozen (Kikuchi et al. 1998, Ikeda et al. 2002). Spermatozoa were thawed in TCM 199 (with Earl's salts; Gibco) adjusted to pH 7.8, and centrifuged for $2 \mathrm{~min}$ at $600 \mathrm{~g}$. The sperm pellet was resuspended in pig fertilization medium (pig FM; Suzuki et al. 2002) supplemented with $5 \mathrm{mg} / \mathrm{ml}$ BSA (Fraction V; Sigma). Spermatozoa were then sonicated for $1 \mathrm{~min}$ to isolate the sperm heads. In man, DTT is known to promote decondensation of sperm chromatin only after the sperm plasma membrane has been made permeable (Reyes et al. 1989). Therefore, before DTT treatment, the spermatozoa were washed in pig FM supplemented with the nonionic detergent TX-100 at $1 \%(\mathrm{v} / \mathrm{v})$ at room temperature to remove the sperm membrane. This treatment exposed the sperm nuclei directly to DTT. The sperm were then incubated at $37^{\circ} \mathrm{C}$ in pig FM supplemented with $5 \mathrm{mM}$ DTT (Wako Pure Chemical Industries, Osaka, Japan). After incubation, the spermatozoa were washed in pig FM without DTT. To determine the appropriate time for sperm incubation, we conducted a preliminary experiment (Fig. 1). We treated the sperm with DTT for 1 min and immediately injected them, still with their original morphology, into mature oocytes.

\section{Procedure of sperm head injection}

Sperm heads were injected as described previously (Nakai et al. 2003). About 20 oocytes were transferred into a 
A

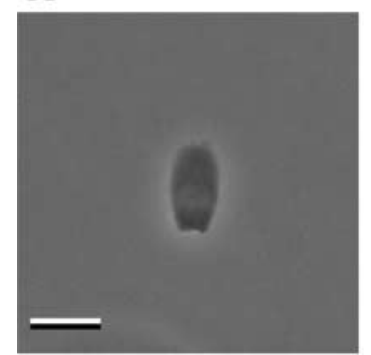

B

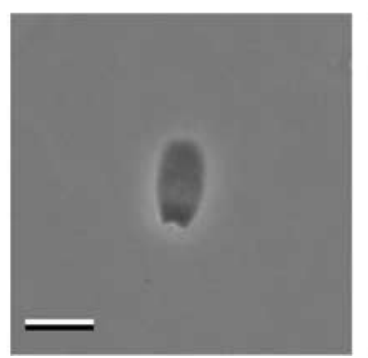

$\mathrm{C}$

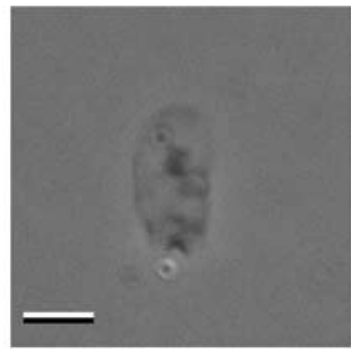

Figure 1 Morphologic changes in sperm heads after treatment with 1\% Triton X-100 and 5 mM dithiothreitol (T + D). (A) untreated sperm head, (B) sperm head immediately (1 min) after $\mathrm{T}+\mathrm{D}$ treatment, and (C) sperm head treated with $\mathrm{T}+\mathrm{D}$ for 1 min and incubated subsequently in $\mathrm{T}+\mathrm{D}-$ free medium at $37^{\circ} \mathrm{C}$ for $1 \mathrm{~h}$. Scale bar: $5 \mu \mathrm{m}$.

$20 \mu \mathrm{l}$ drop of injection solution. The solution consisted of modified IVC-PyrLac-Hepes, which was a modified NCSU-37 without glucose but supplemented with $0.17 \mathrm{mM}$ sodium pyruvate, $2.73 \mathrm{mM}$ sodium lactate, $4 \mathrm{mg} / \mathrm{ml}$ BSA, $50 \mu \mathrm{M} \quad \beta$-mercaptoethanol (IVC-PyrLac) (Kikuchi et al. 2002) and $20 \mathrm{mM}$ Hepes, of which the osmolarity was adjusted to 285 mOsm. A solution containing mature oocytes was placed on the cover of a plastic dish (Falcon 35-1005; Becton Dickinson, Franklin Lakes, NJ, USA). A small volume $(0.5 \mu \mathrm{l})$ of the sonicated sperm head suspension was transferred to $2 \mu$ l drops of injection solution with 4\% (w/v) polyvinyl pyrrolidone (MW $360000 ;$ Sigma), which was prepared close to the drops used for the oocytes. All drops had been covered with paraffin oil (Paraffin Liquid; Nakarai Tesque, Kyoto, Japan). A single sperm head was aspirated from the suspension into an injection pipette, and the pipette was moved to the drop containing the oocytes. The sperm head was injected into the ooplasm with a piezo-actuated micromanipulator (PMAS-CT150; Prime Tech Ltd, Tsuchiura, Japan).

\section{Oocyte stimulation}

One hour after injection, the sperm head-injected oocytes were transferred to an activation solution consisting of $0.28 \mathrm{M}$ D-mannitol, $0.05 \mathrm{mM} \mathrm{CaCl}_{2}, 0.1 \mathrm{mM} \mathrm{MgSO}_{4}$ and $0.1 \mathrm{mg} / \mathrm{ml} \mathrm{BSA}$, and washed once. They were then stimulated with a direct current pulse of $1.5 \mathrm{kV} / \mathrm{cm}$ for $20 \mu \mathrm{s}$ with a somatic hybridizer (SSH-10; Shimadzu, Kyoto, Japan).

\section{In vitro culture of sperm head-injected oocytes}

Sperm head-injected oocytes before and after electrical stimulation were cultured in vitro. Two types of in vitro culture (IVC) medium were prepared (Kikuchi et al. 2002). The first was IVC-PyrLac. The second contained $5.55 \mathrm{mM}$ glucose, as originally reported in NCSU-37 (Petters \& Wells 1993), and also $4 \mathrm{mg} / \mathrm{ml}$ BSA and $50 \mu \mathrm{M} \beta$-mercaptoethanol (IVC-Glu). IVC-PyrLac was used from day 0 (the day of injection and electrical stimulation was defined as day 0 ) to day 2 . The medium was changed once to
IVC-Glu at day 2 and used for subsequent culture. IVC was carried out at $38.5^{\circ} \mathrm{C}$ under $5 \% \mathrm{O}_{2}$.

\section{Assessment of fertilization and embryonic development}

The sperm-injected oocytes and cultured embryos were mounted on glass slides and fixed in 25\% (v/v) acetic acid in ethanol, stained with $1 \%(\mathrm{w} / \mathrm{v})$ orcein in $45 \%(\mathrm{v} / \mathrm{v})$ acetic acid, and examined under a phase-contrast microscope. We conducted the examination at $0-10 \mathrm{~h}$ after a treated or untreated sperm head had been injected and then the oocytes stimulated or unstimulated. A total of 44 experimental groups were evaluated: three replicated trials using 37-138 oocytes were carried out for each group.

\section{Statistical analysis}

Fertilization status (percentages of decondensed sperm heads, recondensed sperm heads, and male pronucleus formation) and morphologic changes in sperm nuclei (transformation to metaphase chromosomes) were scored. Embryonic development to the blastocyst stage (rate of blastocyst formation and mean number of cells per blastocyst) was evaluated. The percentage data were arcsine transformed (Snedecor \& Cochran 1989). All the data were subjected to analysis of variance (ANOVA) by the general linear model procedure of the Statistical Analysis System (SAS Institute, Cary, NC, USA) and were then analyzed by Duncan's multiple-range test.

\section{Experiment 1}

We examined the effects of $1 \%$ TX-100 and $5 \mathrm{mM}$ DTT treatment ( $T+D$ treatment), with or without oocyte stimulation, on morphologic changes in the injected sperm. Some of the oocytes in both the $\mathrm{T}+\mathrm{D}$-treated or untreated groups were fixed every $1 \mathrm{~h}$ after the injection $(0-10 \mathrm{~h}$ after injection) in whole-mount preparations and stained, and the status of the injected sperm heads was evaluated. The sperm heads were categorized into the following five states: 1. condensation (Fig. 2A); 2. decondensation (Fig. 2B); 3. recondensation into a mass (Fig. 2C); 4. pronucleus formation, identified by the presence of at 

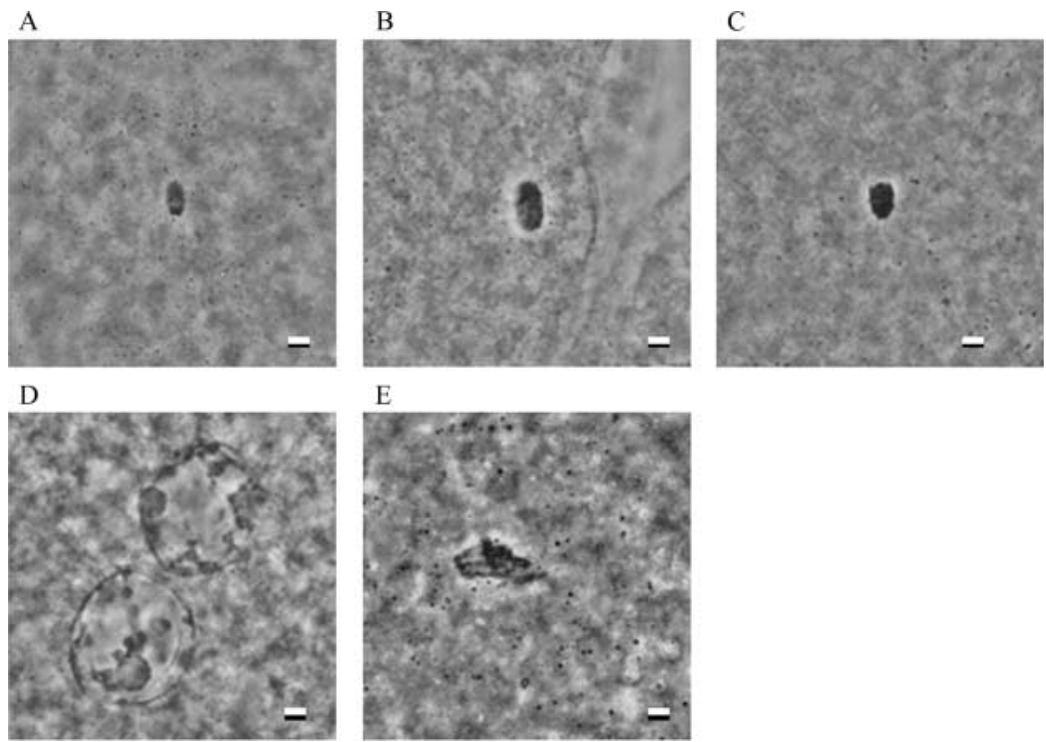

E

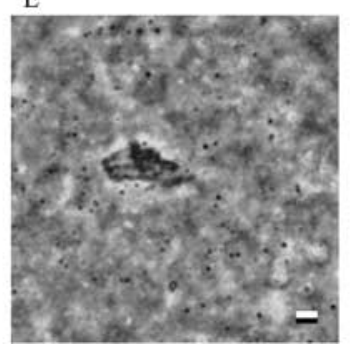

Figure 2 Morphologic changes in sperm heads after injection into mature porcine oocytes. Sperm heads after injection were categorized into five states: (A) condensation, (B) decondensation, (C) recondensation, (D) pronucleus formation, and (E) metaphase chromosome formation. Oocytes were injected with sperm heads treated with Triton X-100 and dithiothreitol (T + D), not stimulated with an electric pulse, and fixed at $6 \mathrm{~h}(\mathrm{~A}-\mathrm{D})$ or $7 \mathrm{~h}(\mathrm{E})$ after injection. Scale bar: $5 \mu \mathrm{m}$.

least two pronuclei (Fig. 2D); and 5. metaphase-like chromosome formation (Fig. 2E). Rates of decondensation, recondensation and pronucleus formation, and the presence of metaphase-like chromosomes were also assessed together as the rate of 'post-condensation'.

\section{Experiment 2}

We evaluated the effects of electrical stimulation and sperm treatment with or without $\mathrm{T}+\mathrm{D}$ on the subsequent development of oocytes after injection of sperm heads. On day 6, the cultured oocytes were fixed and stained, and the percentage of blastocysts and mean numbers of cells per blastocyst were evaluated.

\section{Results}

\section{Experiment 1}

Time-dependent changes in the rates of condensation, decondensation, recondensation, pronuclear formation and metaphase-like chromosome formation, as well as post-condensation in $\mathrm{T}+\mathrm{D}$-treated or untreated sperm heads, with or without oocyte stimulation after injection, are shown in Fig. 3. The results of ANOVA (F-values and significance levels) of the changes in sperm nuclear morphology are shown in Table 1.

\section{Condensation}

Regardless of whether or not the oocyte had been electrically stimulated, a decrease in the percentage of condensed sperm nuclei was recognized in both the $T+D$ treated and untreated groups (Fig. 3A and B). There was a rapid drop in the percentage just after injection in the $\mathrm{T}+\mathrm{D}$-treated group (to $43.0 \%$ by $1 \mathrm{~h}$ in the unstimulated group and to $46.5 \%$ and $15.0 \%$ by 1 and $2 \mathrm{~h}$ respectively, in the stimulated group). In contrast, the rate of condensation decreased gradually in the untreated group (remaining at $98.8 \%$ and $94.7 \%$ at $1 \mathrm{~h}$ in the unstimulated and stimulated groups respectively). Generally, the rates in the $\mathrm{T}+\mathrm{D}$-treated groups were lower than those in the untreated groups, and this difference was significant in terms of the F-value $(P<0.001)$ for the effect of sperm treatment (Table 1). The percentage condensation also differed significantly $(P<0.001)$ according to the period after the injection (Table 1).

\section{Decondensation}

In unstimulated oocytes, the peak rate of sperm nuclear decondensation in the untreated group occurred later $(39.4-44.1 \%, 3-6 \mathrm{~h}$ after injection) than in the $\mathrm{T}+\mathrm{D}$ treated group $(57.0 \%$ and $52.6 \%, 1$ and $2 \mathrm{~h}$ respectively) (Fig. 3C). However, in stimulated oocytes, the peak rate in the untreated group occurred earlier $(42.6-44.1 \%, 2-4 \mathrm{~h})$ - at the same time as that observed in the T + D-treated group (82.5\%, 2 h) (Fig. 3D). Statistical analysis revealed that sperm decondensation was affected significantly $(P<0.001)$ by time elapsed after injection (Table 1$)$.

\section{Recondensation}

When the injected oocytes were not stimulated, the rate of recondensation of sperm heads increased gradually to about $60 \%$ with increasing culture time in both the $\mathrm{T}+\mathrm{D}$-treated and untreated groups (Fig. 3E). Stimulation of oocytes prevented this increase in the rate of sperm 
Stimulation $(-)$
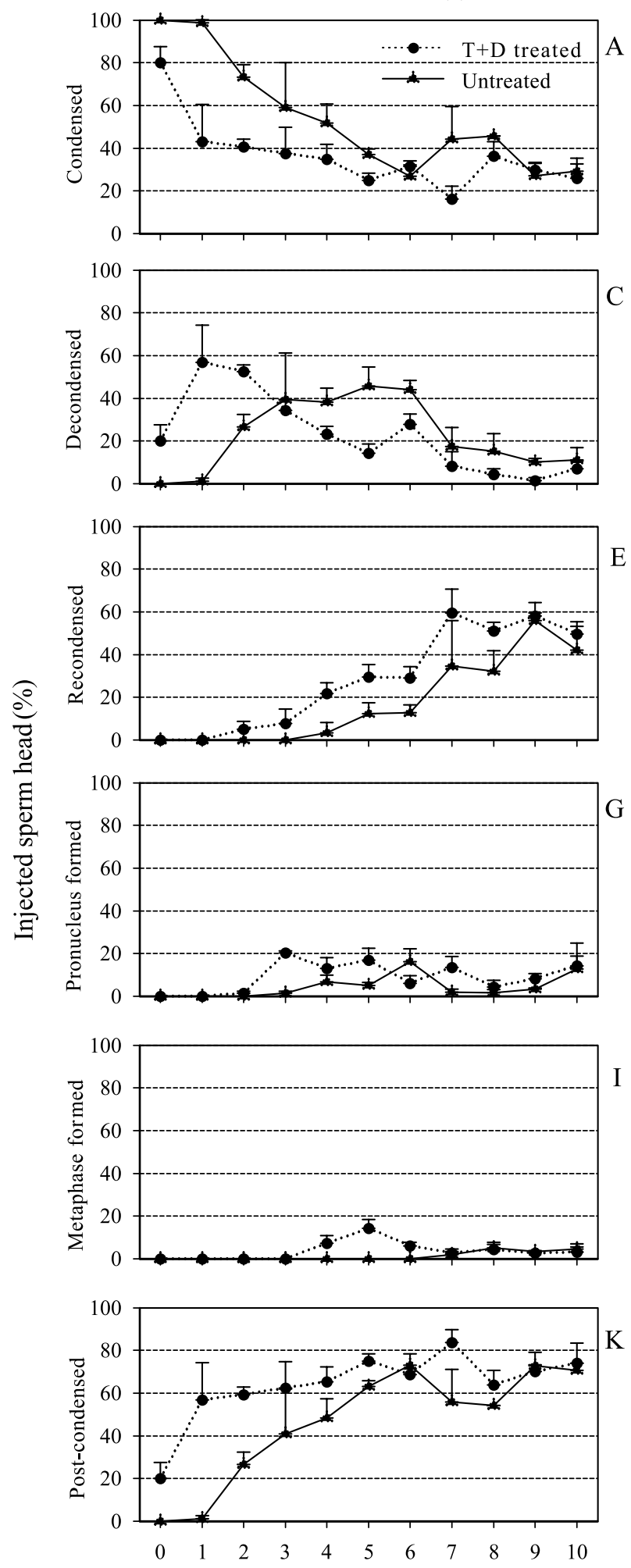

Stimulation $(+)$
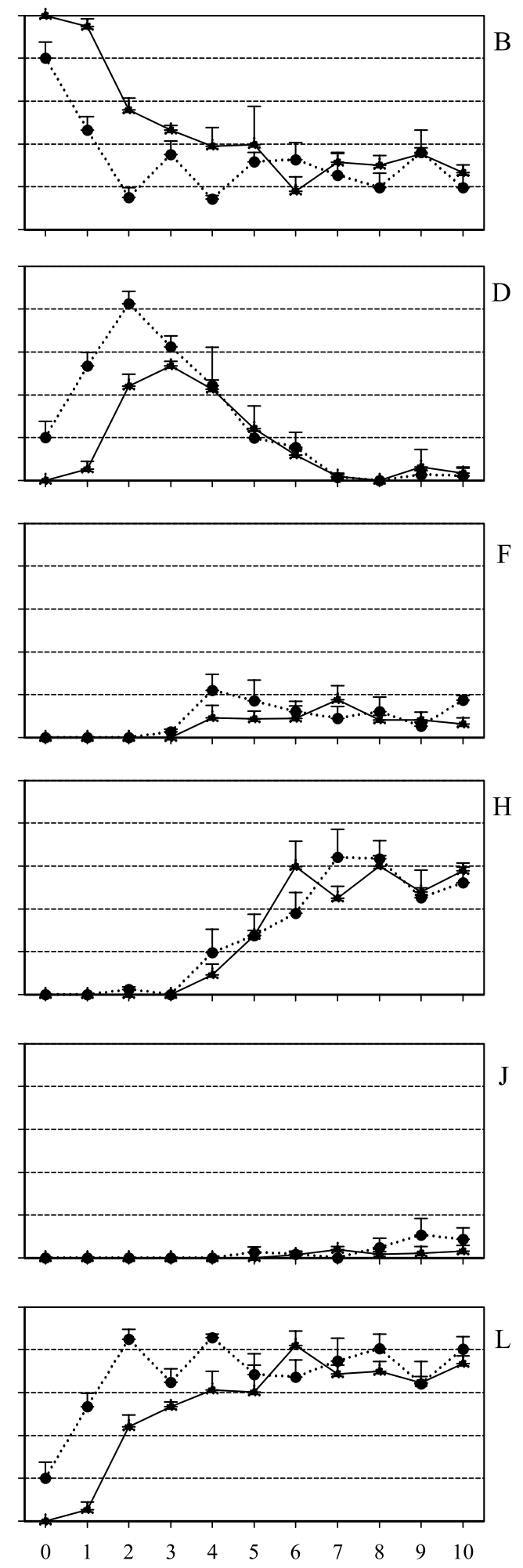

Duration after injection (h)

Figure 3 Time-dependent changes in the rates of condensation ( $\mathrm{A}$ and $\mathrm{B})$, decondensation $(\mathrm{C}$ and $\mathrm{D})$, recondensation (E and $\mathrm{F})$, pronucleus formation $(\mathrm{G}$ and $\mathrm{H})$, metaphase formation ( $\mathrm{I}$ and $\mathrm{J}$ ) and post-condensation ( $\mathrm{K}$ and $\mathrm{L}$ ) of boar sperm heads at every $1 \mathrm{~h}$ after injection. Heads were untreated or pretreated with $1 \%$ Triton X-100 and $5 \mathrm{mM}$ dithiothreitol. Electrical stimulation $(1.5 \mathrm{kV} / \mathrm{cm}, 20 \mu \mathrm{s}$ DC pulse) was applied to the oocytes $1 \mathrm{~h}$ after injection ( $\mathrm{B}, \mathrm{D}, \mathrm{F}, \mathrm{H}, \mathrm{J}$ and $\mathrm{L}$ ) or was not applied (A, C, E, G, I and K). Mean percentages \pm S.E.M. are presented. 
Table 1 ANOVA of morphologic changes of sperm nuclei after sperm head injection in pig.

\begin{tabular}{|c|c|c|c|c|c|c|c|}
\hline \multirow[b]{2}{*}{ Source } & \multirow[b]{2}{*}{ DF } & \multicolumn{6}{|c|}{ F-value } \\
\hline & & Condensation & Decondensation & Recondensation & $\begin{array}{l}\text { Pronuclear } \\
\text { formation }\end{array}$ & $\begin{array}{l}\text { Metaphase-like } \\
\text { chromosome formation }\end{array}$ & Post-condensation \\
\hline Stimulation $^{\mathrm{a}}$ & 1 & 2.67 & 0.03 & $39.73^{* * *}$ & $43.90 * * *$ & 1.40 & 3.17 \\
\hline $\begin{array}{l}\text { Sperm treatment } \\
\text { before injection }\end{array}$ & 1 & $42.85^{* * *}$ & 2.39 & $8.85 * *$ & 1.19 & $7.21 * *$ & $31.61 * * *$ \\
\hline $\begin{array}{l}\text { Culture period } \\
\text { after injection }\end{array}$ & 10 & $22.52 * * *$ & $12.23 * * *$ & $9.36 * * *$ & $9.02 * * *$ & $3.36 * *$ & $13.38^{* * *}$ \\
\hline Error & 119 & & & & & & \\
\hline
\end{tabular}

${ }^{\text {a }}$ Sperm-injected oocytes were stimulated with or without electrical pulse at $1 \mathrm{~h}$ after the sperm head injection.

${ }^{\mathrm{b}}$ Sperm were treated with or without Triton X-100 and dithithreitol.

${ }^{\mathrm{c}}$ Oocytes after the sperm head injection were cultured for $0-10 \mathrm{~h}$, fixed, stained and examined.

$* * P<0.01 ; * * * P<0.001$.

head recondensation (to less than $20 \%$ ) during all culture periods in both sperm treatment groups (Fig. 3F). Statistical analysis revealed that all three main effects were significant $(P<0.01)$. Both electrical stimulation and culture duration had very significant effects $(P<0.001)$; the F-value for stimulation was particularly high, meaning that stimulation was more important than the other two effects for sperm chromatin recondensation (Table 1).

\section{Pronucleus formation}

The rates of pronucleus formation were increased $4 \mathrm{~h}$ after stimulation (to about $40-60 \%$ ) in the $\mathrm{T}+\mathrm{D}$-treated and untreated groups (Fig. 3H). However, when injected oocytes were not stimulated, the rates in both groups were not increased and remained at the basal level (less than $20 \%$ ) for the whole culture period (Fig. 3G). Statistical analysis revealed that the differences in the two effects (electrical stimulation and culture duration) were significant $(P<0.001)$; the F-value for stimulation was quite high, indicating that stimulation was a more important factor in pronucleus formation (Table 1).

\section{Metaphase-like chromosome formation}

The rate of transformation to metaphase-like chromosomes in sperm nuclei was less than $15 \%$ in all experimental groups over the whole culture period (Fig. $3 \mathrm{I}$ and J). Statistical analysis revealed that the effects of sperm treatment and culture period were significant $(P<0.01)$ (Table 1).

\section{Post-condensation}

Generally, the rates of post-condensation in the $\mathrm{T}+\mathrm{D}$ treated groups were higher than in the untreated groups (Fig. 3K and L). Significance was detected in terms of F-values $(P<0.001)$ for both sperm treatment and duration of culture (Table 1).

\section{Experiment 2}

Table 2 shows the rates of blastocyst formation and the average numbers of cells per blastocyst after injection of $\mathrm{T}+\mathrm{D}$-treated or untreated sperm heads with or without electrical stimulation of oocytes. When the oocytes from both the $\mathrm{T}+\mathrm{D}$-treated and untreated groups were stimulated by an electrical pulse, they showed significantly higher blastocyst formation rates $(P<0.01 ; 24.8 \%$ and $27.1 \%$ respectively) than did unstimulated oocytes from both sperm treatment groups $(1.1 \%$ and $4.1 \%$ respectively). No significant difference in the rate of blastocyst formation was detected between the $\mathrm{T}+\mathrm{D}$ and untreated groups. Moreover, there were no significant differences among any of the groups in the mean numbers of cells per blastocyst (14.0-29.4 cells).

\section{Discussion}

Adenot et al. (1991) reported that in mice a sperm head in the ooplasm decondenses just after penetration and then slowly recondenses; it then forms the male pronucleus.

Table 2 Effects of treatment for sperm heads and stimulate to oocytes that were injected sperm heads on the rate of blastocyst formation and the total cells number of blastocyst in pig.

\begin{tabular}{|c|c|c|c|c|}
\hline Treatment & Stimulation & $\begin{array}{c}\text { Total no. of examined } \\
\text { oocytes }\end{array}$ & $\begin{array}{l}\text { No. (\% } \pm \text { S.E.M.) } \\
\text { of blastocyst }\end{array}$ & $\begin{array}{l}\text { Average no. } \pm \text { S.E.M. of cells } \\
\text { in blastocyst }\end{array}$ \\
\hline Triton $\mathrm{X}-100+$ dithiothretiol & + & $\begin{array}{r}105 \\
89\end{array}$ & $26(24.8 \pm 4.8)^{\mathrm{a}}$ & $\begin{array}{l}24.8 \pm 2.4 \\
14\end{array}$ \\
\hline Untreated & $\begin{array}{l}+ \\
-\end{array}$ & $\begin{array}{r}107 \\
97\end{array}$ & $\begin{aligned} 29 & (27.1 \pm 6.1)^{\mathrm{a}} \\
4 & (4.1 \pm 1.5)^{\mathrm{b}}\end{aligned}$ & $\begin{array}{l}29.4 \pm 2.3 \\
23.0 \pm 3.6\end{array}$ \\
\hline
\end{tabular}

Four replicate trials were carried out for each group. Values different superscripts (a,b) are significantly different; $P<0.01$. 
In our present study, almost the same transition of sperm nuclear remodeling was confirmed during porcine ICSI.

Structures around the sperm nucleus are the factors responsible for sperm decondensation during porcine ICSI. It has already been reported that pig sperm have stable membrane properties and that membrane damage is thus beneficial to sperm decondensation during ICSI (Lee \& Yang 2004). Furthermore, the presence of nonnuclear sperm-head structures, such as the acrosome (Katayama et al. 2002), plasma membrane (Lee \& Yang 2004) and perinuclear theca (Sutovsky et al. 2003), reduces the rate of sperm nucleus decondensation. However, the relationship between sperm structures or $\mathrm{S}-\mathrm{S}$ reduction and decondensation or pronucleus formation has not yet been clarified; we therefore injected S-S-modified sperm after treatment with $\mathrm{T}+\mathrm{D}$ and analyzed the time-dependent morphologic changes in the nuclei of the injected sperm. TX-100 affects the acrosomal membrane and also removes the sperm plasma membrane (Perry et al. 1999). Furthermore, DTT can access the sperm nucleus via the perinuclear theca, leading to decondensation of the nucleus (Sutovsky et al. 1997). Treatment with $T+D$ for a short period did not induce complete sperm decondensation (Fig. 1) but did accelerate decondensation after injection. We observed that the rate of sperm nuclear decondensation in the $\mathrm{T}+\mathrm{D}$-treated group rose to about $60 \%$ at $1 \mathrm{~h}$; this peak was shifted forward in comparison with that for the untreated group (Fig. 3C). It has also been reported that, in hamsters, the timing of sperm nuclear decondensation is related to sperm nuclear S-S content (Perreault et al. 1987). Furthermore, the rate of condensed sperm heads in the $\mathrm{T}+\mathrm{D}$-treated groups was lower than in the untreated groups (Fig. 3A and B). However, the pronucleus formation rates did not differ between the groups (Fig. 3G and H). These results clearly suggest that treatment with both TX-100 and DTT accelerates sperm nuclear decondensation after injection but does not affect male pronucleus formation; we therefore need to consider the possibility that pronucleus formation is also affected by an ooplasmic mechanism or mechanisms other than $\mathrm{S}-\mathrm{S}$ reduction in the sperm nucleus. As well as $\mathrm{S}-\mathrm{S}$, it should be noted that weak noncovalent bonds, which can be broken with an agent such as sodium dodecyl sulfate, can also affect the stability of condensed sperm heads (Calvin \& Bedford 1971).

It is interesting that electrical stimulation accelerated the decondensation of injected sperm heads 1 or $2 \mathrm{~h}$ after stimulation (2-3 h after injection) in both the $\mathrm{T}+\mathrm{D}$-treated and untreated groups (Fig. 3D). Although the mechanism of this phenomenon has not yet been clarified, it might involve increases in both intracellular $\mathrm{pH}$ (Shen \& Steinhardt 1978) and the intracellular concentration of calcium (Sun et al. 1992), which are known to occur when oocytes are activated artificially (Ruddock et al. 2001). The changes in $\mathrm{pH}$ and calcium might be related to changes in the activity of enzymes involved in loss of substructure of the perinuclear theca, and thus loss of its stability (Fulton 1984, De Lourdes Juarez-Mosqueda \&
Mujica 1999). Furthermore, activated oocyte cytosol (Cameron \& Poccia 1994) and alkaline pH (Collas 1998) promote decondensation of sperm nuclei.

We observed a time-dependent increase in the percentages of recondensation and pronucleus formation in nonstimulated and stimulated oocytes respectively; these phenomena were observed in both the $\mathrm{T}+\mathrm{D}$-treated and untreated groups (Fig. $3 \mathrm{E}$ and $\mathrm{H}$ respectively). It has been reported that artificial oocyte activation aids ICSI in cows (Chung et al. 2000) and pigs (Lee et al. 2003, Nagashima et al. 2003). This is related to the transition of M-phase promoting factor (MPF) activity in the recipient ooplasm (Kikuchi et al. 1995). The activity of unactivated oocytes gradually decreases during arrest at metaphase II, but it is high enough to maintain the M-phase (Kikuchi et al. 1995, 1999a, 2000). This high MPF activity makes the injected sperm nuclei recondense into a chromatin mass, or they may often change into metaphase chromosomes, as reported in mice (Clarke \& Masui 1987), pigs (Kikuchi et al. 1999b) and cows (Abeydeera \& Niwa 1992). Once the oocyte is activated, MPF activity is reduced to the basal level, resulting in pronucleus formation (Murray et al. 1989). However, some of the activated oocytes show failure of male pronucleus formation, resulting in recondensation or the formation of metaphase-like chromosomes; this could be caused by reactivation of MPF in response to incomplete oocyte activation (Collas et al. 1993, Ito et al. 2004). In fact, in our study, this was reflected in the status of the unstimulated oocytes. Spontaneous oocyte activation was characterized by second polar body emission and retention of the female pronucleus in about $15 \%$ of oocytes; however, the percentage of oocytes at metaphase III (in which the oocyte has a metaphase plate with both a first and a second polar body) was increased to about 30\% $5 \mathrm{~h}$ after injection (data not shown).

Pretreatment of sperm with DTT is useful in bovine ICSI for facilitating pronucleus formation (Rho et al. 1998) and embryo development to the blastocyst stage (Galli et al. 2003). However, our results demonstrated clearly that blastocyst formation is not promoted by the use of $\mathrm{T}+\mathrm{D}$ treated sperm heads for injection in pigs, in agreement with a previous report (Yong et al. 2005). In the light of the fact that acrosome removal before ICSI does not affect either blastocyst formation in vitro or development to term in vivo after transfer to recipients (Nakai et al. 2003), these results suggest that, in pigs, pretreatment of sperm, as by removal of the sperm membranes and reduction of S-S, does not have a marked effect on the development of injected oocytes. From these results, we conclude that the most important factor for development of injected porcine oocytes is not the state of the sperm membranes or $\mathrm{S}-\mathrm{S}$ but activation of the injected oocyte.

In conclusion, pretreatment of sperm with TX-100 and DTT has been shown to bring forward the timing of sperm nuclear decondensation; however, there is still a problem with the course of sperm nuclear decondensation in pig ICSI, because a certain proportion (about 30\%) of con- 
densed sperm remain. Development to the pronuclear and blastocyst stages in vitro was not improved by this pretreatment. Furthermore, we demonstrated clearly that, in pigs, artificial oocyte activation is essential for the development of injected oocytes.

\section{Acknowledgements}

We thank Dr J Kurisaki, Dr M Fahrudin, Dr N W K Karja and $\operatorname{Dr} \mathrm{T}$ Somfai for critical discussion on the present study, and Ms T Aoki and Ms C Terui for technical assistance. The authors declare that there is no conflict of interest that would prejudice the impartiality of this scientific work.

\section{References}

Abeydeera LR \& Niwa K 1992 Ability of in vitro maturing bovine oocyte to transform sperm nuclei to metaphase chromosome. Journal of Reproduction and Fertility 96 565-572.

Adenot PG, Szollosi MS, Geze M, Renard JP \& Debey P 1991 Dynamics of paternal chromatin changes in live one-cell mouse embryo after natural fertilization. Molecular Reproduction and Development 28 23-34.

Calvin HI \& Bedford JM 1971 Formation of disulphide bonds in the nucleus and accessory structures of mammalian spermatozoa during maturation in the epididymis. Journal of Reproduction and Fertility. Supplement 13 65-75.

Cameron LA \& Poccia DL 1994 In vitro development of the sea urchin male pronucleus. Developmental Biology 162 568-578.

Chung JT, Keefer CL \& Downey BR 2000 Activation of bovine oocytes following intracytoplasmic sperm injection (ICSI). Theriogenology 53 1273-1284.

Clarke HJ \& Masui Y 1987 Dose-dependent relationship between oocyte cytoplasmic volume and transformation of sperm nuclei to metaphase chromosomes. Journal of Cell Biology 104 831-840.

Collas P 1998 Cytoplasmic control of nuclear assembly. Reproduction, Fertility, and Development $10581-592$.

Collas P, Sullivan EJ \& Barnes FL 1993 Histone H1 kinase activity in bovine oocytes following calcium stimulation. Molecular Reproduction and Development 34 224-231.

De Lourdes Juarez-Mosqueda M \& Mujica A 1999 A perinuclear theca substructure is formed during epididymal guinea pig sperm maturation and disappears in acrosome reacted cells. Journal of Structural Biology 128 225-236.

Fulton A 1984 The cytoskeleton. In Cellular Architecture and Choreography, pp 1-72. Eds WJ Brammar \& M Edidin. London: Chapman and Hall.

Galli C, Vassilive I, Lagutina I, Galli A \& Lazzari G 2003 Bovine embryo development following ICSI: effect of activation, sperm capacitation and pre-treatment with dithiothreitol. Theriogenology $601467-1480$.

Hoshi K, Yanagida K \& Sato A 1992 Pretreatment of hamster oocytes with $\mathrm{Ca}^{2+}$ ionophore to facilitate fertilization by ooplasmic microinjection. Human Reproduction 7 871-875.

Ikeda H, Kikuchi K, Noguchi J, Takeda H, Shimada A, Mizokami T \& Kaneko H 2002 Effect of preincubation of cryopreserved porcine epididymal sperm. Theriogenology 57 1309-1318.

Ito J, Shimada M \& Terada T 2004 Mitogen-activated protein kinase inhibitor suppresses cyclin B1 synthesis and reactivation of $\mathrm{p} 34^{\text {cdc2 }}$ kinase, which improves pronuclear formation rate in matured porcine oocytes activated by $\mathrm{Ca}^{2+}$ ionophore. Biology of Reproduction $70797-804$.

Katagiri C \& Ohsumi K 1994 Remodeling of sperm chromatin induced in egg extracts of amphibians. International Journal of Developmental Biology 38 209-216.
Katayama M, Koshida M \& Miyake M 2002 Fate of the acrosome in ooplasm in pigs after IVF and ICSI. Human Reproduction $\mathbf{1 7}$ 2657-2664.

Keefer CL 1989 Fertilization by sperm injection in the rabbit. Gamete Research 22 59-69.

Kikuchi K, Izaike Y, Noguchi J, Furukawa T, Daen FP, Naito K \& Toyoda Y 1995 Decrease of histone H1 kinase activity in relation to parthenogenetic activation of pig follicular oocytes matured and aged in vitro. Journal of Reproduction and Fertility 105 325-330.

Kikuchi K, Nagai T, Ikeda H, Noguchi J, Shimada A, Soloy E \& Kaneko H 1998 Cryopreservation and ensuing in vitro fertilization ability of boar spermatozoa from epididymides stored at $4{ }^{\circ} \mathrm{C}$. Theriogenology 50 615-623.

Kikuchi K, Naito K, Noguchi J, Shimada A, Kaneko K, Yamashita M, Tojo H \& Toyoda Y 1999a Inactivation of p34 ${ }^{\text {cdc2 }}$ kinase by the accumulation of its phosphorylated forms in porcine oocytes matured and aged in vitro. Zygote 7 173-179.

Kikuchi K, Nagai T, Ding J, Yamauchi N, Noguchi J \& Izaike Y $1999 b$ Cytoplasmic maturation for activation of pig follicular oocytes cultured and arrested at metaphase-I. Journal of Reproduction and Fertility 116 143-156.

Kikuchi K, Naito K, Noguchi J, Shimada A, Kaneko H, Yamashina M, Aoki F, Tojo H \& Toyoda Y 2000 Maturation/M-phase promoting factor: a regulator of aging in porcine oocytes. Biology of Reproduction 63 715-722.

Kikuchi K, Onishi A, Kashiwazaki N, Iwamoto M, Noguchi J, Kaneko H, Akita T \& Nagai T 2002 Successful piglet production after transfer of blastocysts produced by a modified in vitro system. Biology of Reproduction 66 1033-1041.

Kim NH, Shin JS, Kim C, Jun SH, Lee HT \& Chung KS 1999 Fertilization and in vitro development of porcine oocytes following intracytoplasmic injection of round spermatid or round spermatid nuclei. Theriogenology 51 1441-1449.

Kimura Y \& Yanagimachi R 1995 Intracytoplasmic sperm injection in the mouse. Biology of Reproduction 52 709-720.

Kolbe T \& Holtz W 2000 Birth of a piglet derived from an oocyte fertilized by intracytoplasmic sperm injection (ICSI). Animal Reproduction Science 64 97-101.

Kren R, Kikuchi K, Nakai M, Miyano T, Ogushi S, Nagai T, Suzuki S, Fulka J \& Fulka J Jr 2003 Intracytoplasmic sperm injection in the pig: where is the problem? Journal of Reproduction and Development $49271-273$.

Kuretake S, Kimura Y, Hoshi K \& Yanagimachi R 1996 Fertilization and development of mouse ooctes injected with isolated sperm head. Biology of Reproduction 55 789-795.

Lee JW \& Yang X 2004 Factors affecting fertilization of porcine oocytes following intracytoplasmic injection of sperm. Molecular Reproduction and Development 68 96-102.

Lee JW, Tian XC \& Yang X 2003 Failure of male pronucleus formation is the major cause of lack of fertilization and embryo development in pig oocytes subjected to intracytoplasmic sperm injection. Biology of Reproduction 68 1341-1347.

Martin MJ 2000 Development of in vivo-matured porcine oocytes following intracytoplasmic sperm injection. Biology of Reproduction 63 109-112.

Motoishi M, Goto K, Tomita K, Ookutsu S \& Nakanishi Y 1996 Decondensation of bull and human sperm nuclei by dithiothreitol and/or heparin. Journal of Reproduction and Development $\mathbf{4 2}$ $7-13$.

Murray AW, Solomon MJ \& Kirschner MW 1989 The role of cyclin synthesis and degradation in the control of maturation promoting factor activity. Nature 339 280-286.

Nagashima H, Fujimura T, Takahagi Y, Kurome M, Wako N, Ochiai M, Esaki R, Kano K, Saito S, Okabe M \& Murakami H 2003 Development of efficient strategies for the production of genetically modified pigs. Theriogenology 59 95-106.

Nakai M, Kashiwazaki N, Takizawa A, Hayashi Y, Nakatsukasa E, Fuchimoto D, Noguchi J, Kaneko H, Shino M \& Kikuchi K 2003 Viale piglets generated from porcine oocytes matured in vitro and 
fertilized by Intracytoplasmic sperm head injection. Biology of Reproduction 68 1003-1008.

Perreault SD, Naish SJ \& Zirkin BR 1987 The timing of hamster sperm nuclear decondensation and male pronucleus formation is related to sperm nuclear disulfide bond content. Biology of Reproduction 36 239-244.

Perreault SD, Barbee RR \& Slott VL 1988 Importance of glutathione in the acquisition and maintenance of sperm nuclear decondensing activity in maturing hamster oocytes. Developmental Biology 125 181-186.

Perry ACF, Wakayama T, Kishikawa H, Kasai T, Okabe M, Toyoda Y \& Yanagimachi R 1999 Mammalian transgenesis by intracytoplasmic sperm injection. Science 284 1180-1183.

Petters RM \& Wells KD 1993 Culture of pig embryos. Journal of Reproduction and Fertility. Supplement 48 61-73.

Reyes R, Rosado A, Hernandez O \& Delgado NM 1989 Heparin and glutathione: physiological decondensing agents of human sperm nuclei. Gamete Research 23 39-47.

Rho GJ, Kawarsky S, Johnson WH, Kochhar K \& Betteridge KJ 1998 Sperm and oocyte treatments to improve the formation of male and female pronuclei and subsequent development following intracytoplasmic sperm injection bovine oocytes. Biology of Reproduction 59 918-924.

Ruddock NT, Machaty Z, Cabot R \& Prather R 2001 Porcine oocyte activation: differing roles of calcium and $\mathrm{pH}$. Molecular Reproduction and Development 59 227-234.

Seligman J, Kosower NS, Weissenberg R \& Shalgi R 1994 Thioldisulfide status of human sperm proteins. Journal of Reproduction and Fertility 101 435-443.

Shen SS \& Steinhardt RA 1978 Direct measurement of intracellular $\mathrm{pH}$ during metabolic derepression of the sea urchin egg. Nature $272253-254$

Shimada A, Kikuchi K, Noguchi J, Akama K, Nakano M \& Kaneko H 2000 Protamine dissociation before decondensation of sperm nuclei during in vitro fertilization of pig oocytes. Journal of Reproduction and Fertility $120247-256$.

Snedecor GW \& Cochran WG 1989 In Statistical Methods, 8th edn, pp 273-296. Ames, IA: lowa State University Press.

Sun FZ, Hoyland J, Huang X, Mason W \& Moor RM 1992 A comparison of intracellular changes in porcine eggs after fertilization and electroactivation. Development 115 947-956.
Sutovsky P, Oko R, Hewitson L \& Schatten G 1997 The removal of the sperm perinuclear theca and its association with the bovine oocyte surface during fertilization. Developmental Biology 188 75-84.

Sutovsky P, Manandhar G, Wu A \& Oko R 2003 Interaction of sperm perinuclear theca with the oocyte: implications for oocyte activation, anti-polyspermy defense, and assisted reproduction. Microscopy Research and Technique $61362-378$.

Suzuki K, Asano A, Eriksson B, Niwa K, Nagai T \& Rodrigues-Martinez H 2002 Capacitation status and in vitro fertility of boar spermatozoa: effects of seminal plasma, cumulus-oocyte-complexesconditioned medium and hyaluronan. International Journal of Andrology 25 84-93.

Tesarik J \& Sousa M 1995 More than 90\% fertilization rates after intracytoplasmic sperm injection and artificial induction of oocyte activation with calcium ionophore. Fertility and Sterility $\mathbf{6 3}$ $343-349$.

Yanagimachi R 1994 Mammalian fertilization. In The Physiology of Reproduction, 2nd edn, pp 189-317. Eds E Knobil \& JD Neill. New York: Raven Press.

Yong HY, Hong JY, Kang SK, Lee BC, Lee ES \& Hwang WS 2005 Sperm movement in the ooplasma, dithiothreitol pretreatment and sperm freezing are not required for the development of porcine embryos derived from injection of head membrane-damaged sperm. Theriogenology 63 783-794.

Yoshida M, Ishigaki K, Nagai T, Chikyu M \& Pursel GV 1993 Glutathione concentration during maturation and after fertilization in pig oocytes: relevance to the ability of oocytes to form male pronucleus. Biology of Reproduction 49 89-94.

Zirkin BR, Perreault SD \& Naish SJ 1989 Formation and function of the male pronucleus during mammalian fertilization. In The Molecular Biology of Fertilization, pp 91-114. San Diego, CA: Academic Press.

Received 11 October 2005

First decision 15 November 2005

Revised manuscript received 9 December 2005

Accepted 9 December 2005 